\title{
RETROSYNTHESIS FAVOURS e-CHEMISTRY
}

\section{C.K. Patil1, V. H. Masand ${ }^{2}$, K. N. Patil ${ }^{3}$}

${ }^{1}$ Department of Chemistry, L.A.D. College for Women, Nagpur. 2,3Post-Graduate Department of Chemistry, VidyaBharati College,

Amravati-Maharashtra (India) - 444602

Author for correspondence: vijaymasand@gmail.com or vijaymasand@rediffmail.com Phone no.: 0091-9822467909

\section{Abstract:}

In present work, use of retrosynthesis, its advantages and mathematical modeling used are discussed along with anatomy of retrosynthetic analysis. Hendrickson's equation is found to be extremely useful for selection of good synthetic route for the synthesis of TM, and minimizes "trial and errors".

\section{Graphical abstract:}



Keywords: Retrosynthesis, Disconnection approach, Mathematical modeling

Introduction: Solving synthetic challenges and problems always give immense pleasure to every organic chemist, who wants to synthesize the target molecule by possible shortest route. During the design emphasis on 'easy, efficient, economical and eco-friendly' synthesis of target molecule is thought i.e. 'e-chemistry' route to synthesize such molecules.

These goals can be achieved quite expediently by using modern synthetic methodology of 'Retrosyntheis'. Retrosynthesis was introduced, 
extensively used and popularized by E. J. Corey, for which, he received 'Noble Prize' in chemistry.

Wipke $^{1}$ defined it as "the process of breaking down a target molecule into readily available starting material by means of imaginary breaking of bonds (disconnections) and by the conversion of one functional group to another by efficient chemical reactions (FGI)”.

Frequently, researchers derive linear as well as convergent paths for a target molecule with the help of retrosynthesis, by considering various parameters like cost, efficiency, timing, etc (figure 1). and finally accept one of the path for synthesis. The same method was applied to the following molecules, whereby, the retrosynthetic analysis, interestingly, gave linear and convergent paths with same no. of steps, same reagents and timing (figure 2).

\section{Methodology:}

The problem: which route to choose? Was solved by using mathematical modeling proposed by Hendrickson ${ }^{2}$. The formula is as follows;

$$
\mathrm{W}=\Sigma \eta_{\mathrm{i}} \mathrm{X}^{\mathrm{li}}
$$

Where $\eta_{i}$ is no. of skeletal carbons in each piece and $X$ is reciprocal of the average yield for each step. The whole quantity $\mathrm{X}^{\mathrm{li}}$ is related to no.of steps 1 . The values of $\mathrm{X}$ for a reaction path of 15 steps are listed in table 1. The above formula is based on the assumption that there is no refunctionalisation in the synthesis. Lower the value of $\mathrm{W}$, better is the path.

\begin{tabular}{llll}
\hline No. of steps $(1)$ & Actual $\left(\mathrm{X}^{\mathrm{a}}\right)$ & Approximate $\left(\mathrm{X}^{\mathrm{a}}\right)$ & Yield \\
\hline 1 & 1.25 & 1.25 & 80 \\
2 & 1.56 & 1.5 & 64.1 \\
3 & 1.95 & 2 & 51.3 \\
4 & 2.44 & 2.5 & 41.0 \\
5 & 3.05 & 3 & 32.8 \\
6 & 3.81 & 4 & 26.2 \\
7 & 4.77 & 5 & 21.0 \\
\hline a $\mathrm{X}$ is reciprocal of percentage yield
\end{tabular}

Table 1: Value of $x$ and yield of syntheses for 1 steps 


\section{Results and discussions:}

The synthetic tree for linear and convergent path and retrosynthetic analysis are shown in figure 1 and 2 . The values of $\mathrm{W}$ were calculated for both the linear and convergent paths. For molecules 1, path a provided $\mathrm{W}=16.25$ where as path $\mathrm{b}$ gave $\mathrm{W}=35.75$ thus the better choice being path a, was chosen to prepare $\mathbf{1}$.

On the same basis, for molecule 2 , path a furnished $\mathrm{W}=56.25$ and path $\mathrm{b}$ gave $\mathrm{W}=82.25$ Thus path a being more attractive choice. The molecules were synthesized 6,7 by both the paths, finally confirming above analysis.

\section{Conclusion:}

In conclusion, whenever there is a tie up between linear and convergent path at parameter level, mathematical modeling could be a good tool to solve the problem for the route decision, especially for giant complex molecules. Before giving any decisive conclusion, we deem that a detailed study and knowledge of various aspects like cost, percentage yield, side reactions, timing etc must be under consideration and real experimental work has no alternative.

Acknowledgement: We thank Dr. D.T Mahajan for helpful discussions throughout the work.

\section{References:}

W.T. Wipke, and E. J. Corey, (1969). Science, 166, 178

C.L. Willis, and M. Willis, (1995). Oxford Science Publication, Chapter 4, 34-38

S. Hanessian, J. Franco, B. Larrouche, (1990). Pure Appl. Chem., 62, 1887

J.R. Hendrickson, (1986) Acc. Chem. Res., 19, 274 
J.R. Hendrickson, (1977). J. Am. Chem. Soc., 99, 5439

B.M. Desai, P.R. Desai, R.D. Desai, (1960). J. Ind. Chem. Soc., 37, 53

Paper in communication

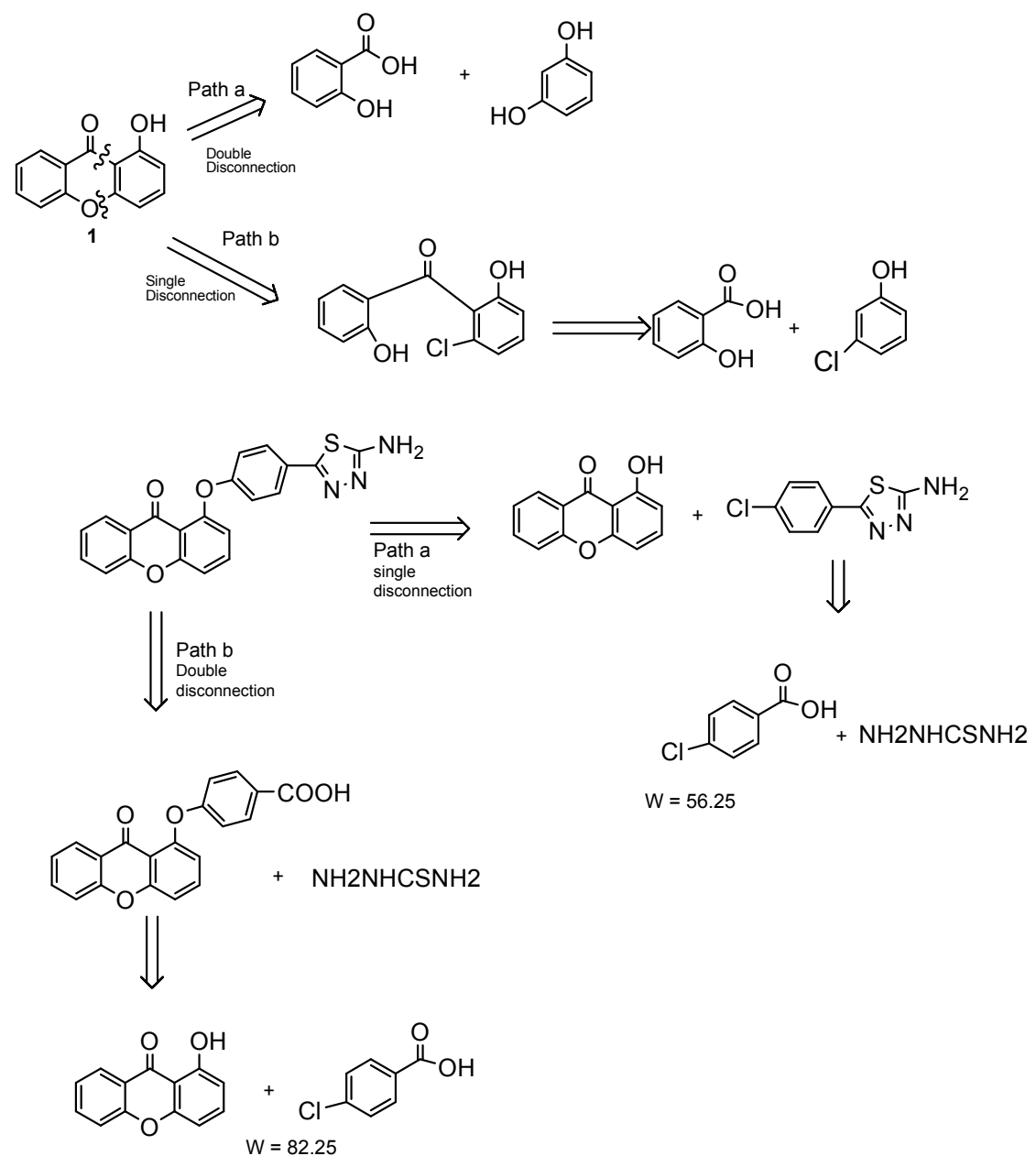

Figure 1: Retrosynthesis of Some heterocyclic molecules

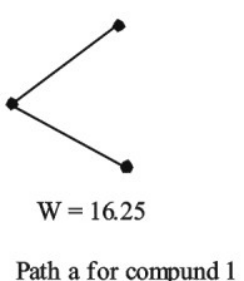

Path a for compund 1

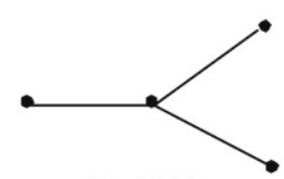

$\mathrm{W}=35.75$

Path $\mathrm{b}$ for compound 1

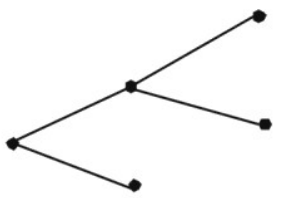

$\mathrm{W}=56.25$

Path a for compund 2

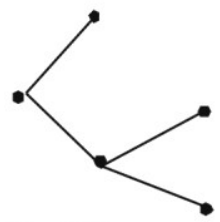

$\mathrm{W}=82.25$

Path $\mathrm{b}$ for compound 2

Figure 2: Synthetic trees for compound 1 and 2 